\title{
Born Again: Recurrent Hepatic Encephalopathy in an Adult without Cirrhosis
}

\author{
Tom George ${ }^{1}$ Sasidharan Rajesh ${ }^{1} \quad$ Cyriac Abby Philips ${ }^{2}$
}

\author{
${ }^{1}$ Department of Interventional Radiology, Cochin Gastroenterology \\ Group, Ernakulam Medical Centre, Kochi, Kerala, India \\ ${ }^{2}$ The Liver Unit, Cochin Gastroenterology Group, Ernakulam Medical \\ Centre, Kochi, Kerala, India
}

Address for Correspondence Tom George, MD, DNB, FVIR, Chalissery House, P.T Road, Chembumukku, Thrikkakara P.O Kochi, Kerala 682021, India (e-mail: mail2dr.tomgeorge@gmail.com).
Abstract
Keywords
- Abernethy malformation
- congenital extrahe- patic portosystemic shunt
- portosystemic shunt

Congenital extrahepatic portosystemic shunt (CEPS) develops between the porto-mesenteric and systemic veins. The splanchnic venous system drains directly to systemic circulation in this condition. We present a case of an adult onset Type II CEPS presenting with hepatic encephalopathy. The patient underwent PARTO (plug-assisted retrograde transvenous obliteration) and had a significant clinical improvement on subsequent follow-up. Our case highlights the importance of imaging and the role of an interventional radiologist in the management of the condition.

A 43-year-old-man developed disorientation while driving, losing control of the car and hit the pedestrian curb side. Evaluation in the emergency department revealed hyperammonemia with coarse echotexture of the liver on ultrasound imaging. He was diagnosed to have hepatic encephalopathy with underlying chronic liver disease and portal hypertension and was referred for further management. On further evaluation, liver tests were normal, magnetic resonance imaging of the brain was within normal limits and liver stiffness was 5.4 (normal $<6 \mathrm{kPa}$ ) excluding a diagnosis of cirrhosis or central nervous system involvement. Contrast-enhanced computed tomography (CECT) imaging revealed a prominent portosystemic shunt (PSS) between the inferior mesenteric vein and the left internal iliac vein and attenuated portal vein without features of chronic liver disease or portal hypertension suggestive of type 2 congenital extrahepatic portosystemic shunt (CEPS, Abernethy malformation; -Fig. 1). The patient underwent plug-assisted retrograde transvenous occlusion (PARTO) of PSS ( - Fig. 2 ) via jugular (right internal jugular vein) access. One month following PARTO procedure, complete thrombosis of PSS was observed with increase in the calibre of the main portal vein and its right branch (-Fig. 3). The patient is back to performing his daily routines. Abernethy malformation is a rare congenital anomaly in which there is direct/indirect communication between the portal and systemic venous

received January 16, 2019 accepted February 17, 2019

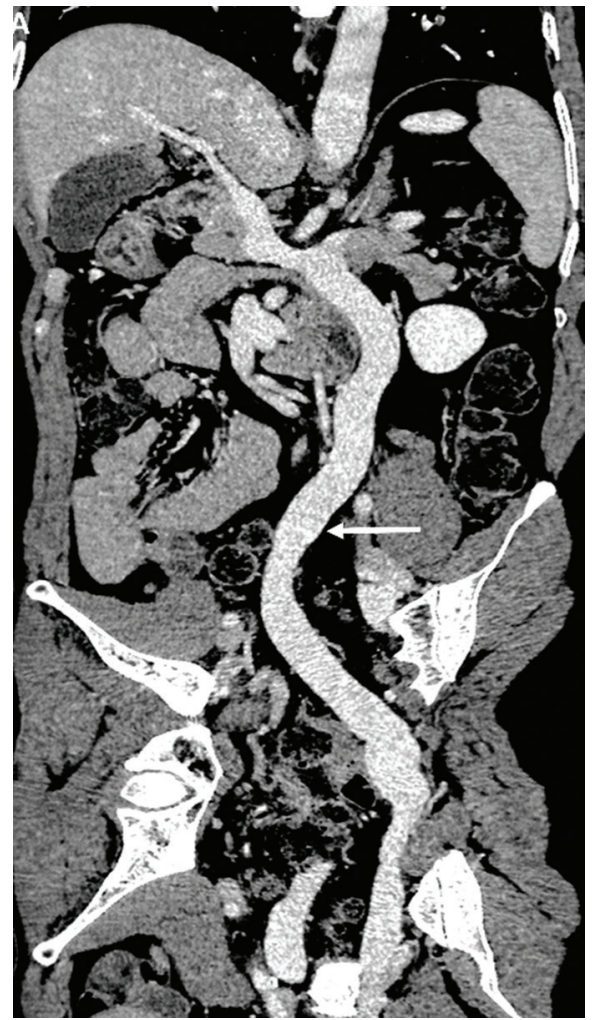

Fig. 1 Contrast-enhanced computed tomography (CECT) reveals large extra-hepatic porto-systemic shunt (arrow) between the inferior mesenteric vein and the left internal iliac vein.
C2019 Indian Society of Gastrointestinal and Abdominal Radiology
License terms

10.1055/s-0039-1686966 


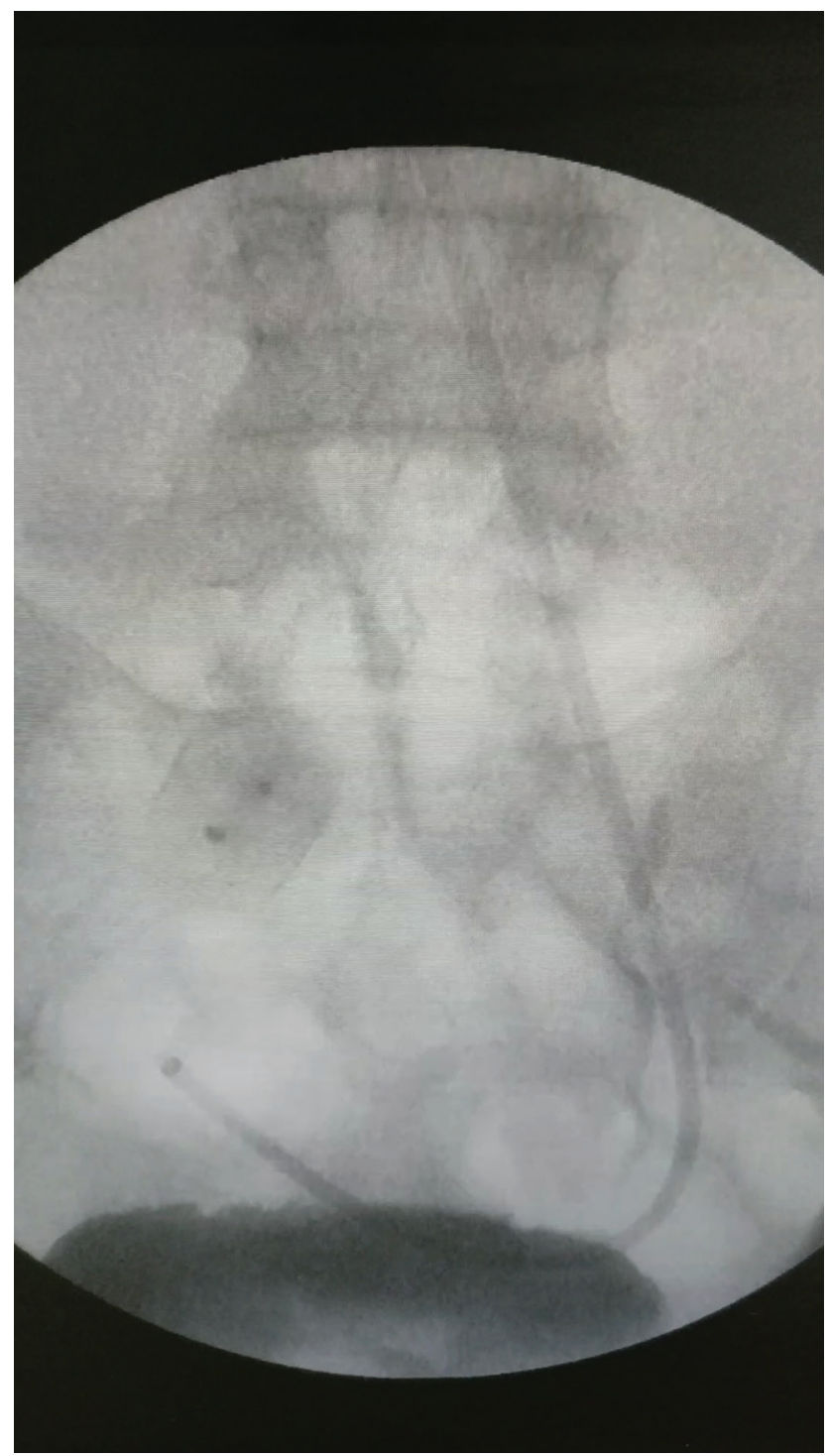

Fig. 2 Fluoroscopy image demonstrating the vascular access sheath coursing through left common and internal iliac vein. Note the type II vascular plug deployed within the shunt.

circulation. Patients present with features related to the shunting of portal blood, associated congenital abnormalities, or hepatic lesions such as hepatoblastoma or focal nodular hyperplasia. Adult onset presentation of CEPS is very rare and for type 2 Abernethy malformation, shunt occlusion by an interventional radiologist is an excellent life-saving treatment option.

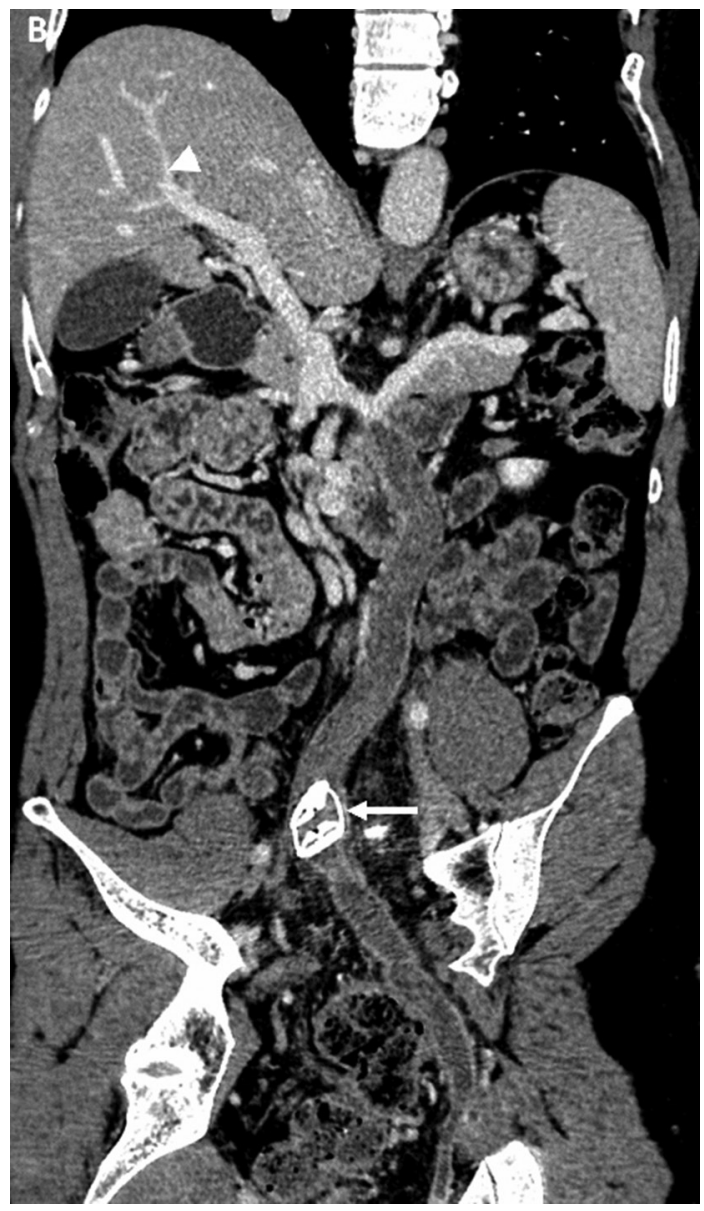

Fig. 3 CECT image taken 1 month after the procedure demonstrating complete thrombosis of the PSS with the vascular plug optimally placed in situ (arrow). Increase in caliber of the main portal vein and its right branch (arrowhead) can also be noted.

\section{Authors' Contributions}

Authors S.R. and C.A.P.: study concept and design; authors S.R. and T.G.: acquisition of data; author C.A.P.: drafting of the manuscript; and authors C.A.P., S.R. and T.G.: critical revision of the manuscript for important intellectual content.

\section{Conflict of Interest}

None declared. 